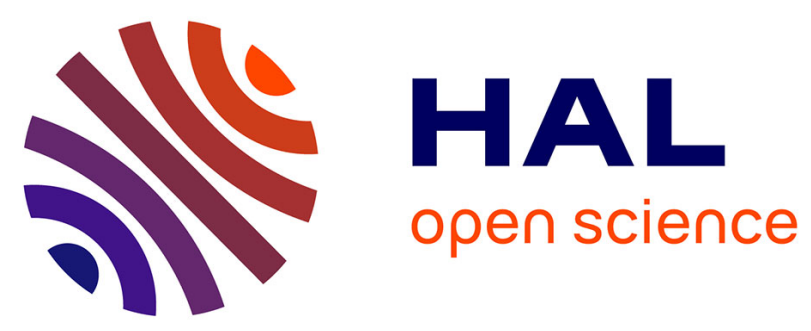

\title{
Degenerate scale for 2D Laplace equation with mixed boundary conditions and comparison with other conditions on the boundary
}

Alain Corfdir, Guy Bonnet

\section{- To cite this version:}

Alain Corfdir, Guy Bonnet. Degenerate scale for 2D Laplace equation with mixed boundary conditions and comparison with other conditions on the boundary. Engineering Analysis with Boundary Elements, 2018, 88, pp.14-25. 10.1016/j.enganabound.2017.12.004 . hal-01695864

\section{HAL Id: hal-01695864 https://hal.science/hal-01695864}

Submitted on 29 Jan 2018

HAL is a multi-disciplinary open access archive for the deposit and dissemination of scientific research documents, whether they are published or not. The documents may come from teaching and research institutions in France or abroad, or from public or private research centers.
L'archive ouverte pluridisciplinaire HAL, est destinée au dépôt et à la diffusion de documents scientifiques de niveau recherche, publiés ou non, émanant des établissements d'enseignement et de recherche français ou étrangers, des laboratoires publics ou privés. 


\title{
Degenerate scale for 2D Laplace equation with mixed boundary condition and comparison with other conditions on the boundary
}

\author{
A. Corfdir ${ }^{\mathrm{a}, *}$, G. Bonnet ${ }^{\mathrm{b}}$ \\ ${ }^{a}$ Laboratoire Navier, École des Ponts, IFSTTAR, CNRS, UPE, UMR 8205, 6/8 avenue Blaise Pascal, Champs-sur-Marne, France \\ ${ }^{\mathrm{b}}$ Laboratoire Modélisation et Simulation Multi-Echelle, Université Paris Est, MSME UMR 8208 CNRS, 5 boulevard Descartes, Marne la Vallée Cedex 77454 France
}

A R T I C L E I N F O

\section{Keywords}

Integral equations

Boundaryelements

Laplace equation

Plane problems

Degenerate scale

Mixed boundary condition

Neumann condition

Dirichlet condition

\begin{abstract}
A B S T R A C T
It is well known that the 2D Laplace Dirichlet problem has a degenerate scale for which the direct boundary integral equation has several solutions. We study here the case of the mixed boundary condition, mainly for the exterior problem, and show that this problem has also one degenerate scale. The degenerate scale factor is a growing function of the part of the boundary submitted to Neumann condition. Different special cases are then addressed: segment, circle and symmetric problems. Some exact values of the degenerate scale factor are given for equilateral triangle and square. The numerical procedure for determining the degenerate scale factor for mixed BC is described. The comparison is made with other kinds of boundary conditions and the consequence of the choice of Green's function when using the Boundary Element Method is studied.
\end{abstract}




\section{Introduction}

Since its early development, the Boundary Element Method (BEM) method has been intensively investigated. Notably, the error of the BEM has been evaluated [1-3] and new numerical improvements are still being suggested using adaptative mesh [4-7], isogeometric boundary element analysis with non-uniform rational B-splines [8-10] and adaptative cross approximation [11] or reducing the singularities of the boundary integral formulation $[12,13]$.

However, the issue of the degenerate scale for 2D problems persists because it is linked to the underlying Boundary Integral Equation (BIE). The existence of a degenerate scale for the Laplace equation with Dirichlet boundary condition (BC) in the plane is well known $[14,15]$ : for a special size of the domain under study, characterized by its scale when compared with all homothetic domains (the "degenerate scale"), the BIE has more than one solution for Dirichlet BC over all the boundary. Numerous results were obtained on the Laplace problem with Dirichlet BC, either from a fundamental point of view, in relation with potential theory [16-23] or for application to BEM [24-34]. Specific investigations for Laplace's problem deal with the way to evaluate numerically the degenerate scale [27,35], to mitigate the numerical issues coming from degenerate scale $[24,26,27,33,36]$ or to deal with special geometrical configurations $[34,37-40]$ or multiply connected problems [29,30,41]. Some of these results were extended to the case of plane elasticity [42-48] and to biharmonic equation [49,50]. For conduction problems, it has been shown that degenerate scale for anisotropic conduction can be obtained from the ones obtained in the case of isotropy [51]. The Laplace problem with Dirichlet BC has been extended to the case where the Green's function corresponds to the half plane [52] and to Robin boundary condition [53]. It is also worthwhile mentioning that the question arises also in dynamics: under special conditions, the degenerate scales arise also in solving the Helmholtz equation [54], because the dynamic singular kernel is asymptotically logarithmic. From a general point of view, degenerate scales appear because of the logarithmic part of the kernels that are involved when solving plane problems using a Boundary Integral Equation, which is the case for conduction, elasticity, elastoplasticity,...

The mixed BC (i.e. when a part of the boundary is at Dirichlet BC while the other part is at Neumann BC) is the main contribution of the present paper. It has been investigated in [19,28], for the interior problem uniquely. It was shown that if the logarithmic capacity $[16-18,23,31,55]$ is equal to 1 (or equivalently if the Dirichlet problem is at its degenerate scale), the interior mixed problem is at a degenerate scale. The existence and uniqueness of the solution of the integral equations for the interior problem are proved in [56] with the condition that the diameter of the domain is less than 1 ; this paper also contains an extensive bibliography of early works on mixed boundary problems.

The aim of this paper is to complete the results on degenerate scale by providing a thorough study of the case of mixed BC. We focus on the direct method of BIE (see for example [57]). In a first step, we recall the main results in the case of boundary conditions which are of the same kind over all the contour. Next, we complete the known results

* Corresponding author.

E-mail addresses: corfdir@cermes.enpc.fr (A. Corfdir), Guy.Bonnet@univ-mlv.fr (G. Bonnet). 
in the case of the interior boundary value problem by showing that the degenerate scale for Dirichlet $\mathrm{BC}$ is the unique degenerate scale for the interior problem with mixed BC. Then, we address the exterior problem. One difference compared with the case of the interior problem is that the non unique solution of the BIE leads to a non null solution of the boundary problem via the representation formula. There is a unique degenerate scale for this exterior mixed problem and the degenerate scale factor increases when the part of the boundary submitted to Neumann $\mathrm{BC}$ increases. The special case of segments is dealt with. Then some special cases are solved using conformal mappings and symmetries, leading to exact solutions for some cases of equilateral triangle and square. The numerical procedure for obtaining the degenerate scale is provided and finally, we compare the degenerate scale for mixed BC with other cases where the BC is the same over all the boundary: Dirichlet, Robin, Neumann.

\section{Principal results on degenerate scales for $\mathrm{BC}$ of the same type over the contour}

This section reminds shortly the most important results on degenerate scales in the case of Dirichlet, Robin and Neumann BC. It aims to prepare the study of mixed BC which is an analogous issue, needing often similar methods, and to allow the final comparison for all boundary conditions in Section 11.

\subsection{Dirichlet $B C$}

The case of Dirichlet BC over all the contour has been the object of numerous studies. The degenerate scale is related to the nature of the BIE in the case of plane problems, which contains the integral operator over the boundary $\Gamma$

$F_{q}(x)=\int_{\Gamma} q(y) G(x, y) \mathrm{d} S_{y}$,

Where $G$ is the Green function for the plane, $G=\frac{-1}{2 \pi} \ln (\|x-y\|)$. The problem is to find the function $q$ when the function $F_{q}(x)$ is known over $\Gamma$.

$F_{q}(x)$ is known from the boundary condition in the case of Dirichlet $\mathrm{BC}$, either in the case of direct or indirect formulation of the BIE, while the meaning of $q$ differs between these two formulations. In the case of the Direct formulation of BIE, it is given by:

$F_{q}(x)=\frac{1}{2} u(x)+\int_{\Gamma} u(y) \frac{\partial G(x, y)}{\partial n(y)} \mathrm{d} S_{y}$,

In this formulation, $q$ is the unknown boundary normal flux.

The degenerate scale is obtained by studying all domains corresponding to homothetic contours $\rho \Gamma$ of $\Gamma$. It was shown that this operator can be null for non null values of $q$ and a specific boundary $\rho_{0} \Gamma$ which is homothetic to $\Gamma$ by a factor $\rho_{0}$. This specific value of $\rho_{0}$ is the degenerate scale factor. This factor is related to the logarithmic capacity $C$ $[16-18,23]$ by $\rho_{0}=1 / C$. This result is important since the logarithmic capacity of numerous kinds of contours is known, that allows to find easily the degenerate scale, a domain being at the degenerate scale if its logarithmic capacity is null. The search of degenerate scale does not involve the normal to the boundary. As a consequence, interior and exterior problems with Dirichlet BC related to the same boundary correspond to the same degenerate scale.

The existence of degenerate scales has an important consequence when using the BEM, which rests on the discretized version of the boundary integral equation, i.e. the linear system:

$[H][u]=[G][q]$

where $[u]$ contains the values of the searched harmonic potential at nodes located at the boundary and $[q]$ the values of the normal gradient of the potential at the same nodes. The numerical degenerate scale corresponds to the size of the domain for which the matrix $[G]$ is singular. The consequence of the existence of a degenerate scale is that the matrix $[G]$ becomes badly conditioned for domains whose scales are near the one of the boundary at the degenerate scale [28].

\subsection{Convection type or Robin BC}

A convection type $\mathrm{BC}$ has the form :

$\frac{\partial u}{\partial n}=-t\left(u-u_{0}\right)$,

where $u_{0}$ is given over the boundary and $t$ is a positive physical constant related to the convection coefficient and to the conductivity. Even if this condition is well known in the case of thermal condition, it can be used in other physical cases related to conduction: it corresponds to the existence of a very conductive thin layer over the boundary. Assuming that this boundary condition is applied over all the boundary, the boundary integral equation over $\Gamma$ can be written as:

$\frac{1}{2} u(x)+\int_{\Gamma} G(x, y) t u(y) \mathrm{d} S_{y}+\int_{\Gamma} \frac{\partial G}{\partial n_{y}} u(y) \mathrm{d} S_{y}=\int_{\Gamma} G(x, y) t u_{0}(y) \mathrm{d} S_{y}$.

If the domain bounded by $\Gamma$ is at a degenerate scale for this $\mathrm{BC}$, it means that the operator on the left hand side has a non-null solution for a null value of $u_{0}$. It means that the problem for $u_{0}=0$ in the convection condition has a non null solution. This corresponds to the Robin condition, $\mathrm{t}$ being the Robin constant.

The main results concerning the degenerate scale for this problem can be found in [53]. In this paper, it has been shown that, for the interior problem, the degenerate scale is the same as for the Dirichlet problem. However, for the exterior problem, the degenerate scale is now related to the value of the Robin constant.

\subsection{Neumann $B C$}

In the case of Neumann $\mathrm{BC}$ over all the contour, it is well known that the solution of the interior problem is obtained up to a constant if the compatibility condition $\int_{\Gamma} \frac{\partial u}{\partial n} \mathrm{~d} S_{y}=0$ is satisfied (e.g. [58]). There is always a solution of the exterior problem which is unique up to a constant (e.g. [58]). There is no degenerate scale.

\section{Study of the degenerate scale of interior problem with mixed boundary condition}

As seen previously, a degenerate scale does appear in the case of Dirichlet BC and in the case of convection (Robin) BC. However, in practice, different boundary conditions may be mixed and another very important case is the case when a part of the boundary corresponds to Dirichlet BC and the other to Neumann BC. This mixed BC case will be studied in this section for interior problem and in the following sections 4-10 for exterior problems.

\subsection{Definition of the degenerate scale for interior problem with mixed $B C$}

We consider the following interior Laplace problem:

$\left\{\begin{array}{l}\Delta u=0 \quad x \in \Gamma^{+} ; \\ u(x)=0 \quad x \in \Gamma_{D} ; \\ \frac{\partial u}{\partial n}(x)=0 \quad x \in \Gamma_{N} .\end{array}\right.$ 7]1):

Such a function satisfies the following boundary equations (e.g.

$$
\begin{cases}\int_{\Gamma_{N}} u(y) H(x, y) \mathrm{d} S_{y}-\int_{\Gamma_{D}} q(y) G(x, y) \mathrm{d} S_{y}=0 & x \in \Gamma_{D} \\ \frac{1}{2} u(x)+\int_{\Gamma_{N}} u(y) H(x, y) \mathrm{d} S_{y}-\int_{\Gamma_{D}} q(y) G(x, y) \mathrm{d} S_{y}=0 & x \in \Gamma_{N}\end{cases}
$$$$
\text { with } q=\frac{\partial u}{\partial n}, G(x, y)=-\frac{1}{2 \pi} \ln (|x-y|), H(x, y)=\frac{\partial G(x, y)}{\partial n_{y}} \text {. }
$$ 
We say that the interior problem with Dirichlet condition on $\Gamma_{D}$ and Neumann condition $\Gamma_{N}$ is at the degenerate scale if there exists a non null solution $(u, q)$ of (7) defined on $\Gamma_{N} \times \Gamma_{D}$.

3.2. Behavior of the problem with mixed BC if the whole contour is at the degenerate scale for Dirichlet $B C$

This problem has already been investigated in [19] using complex methods and more recently in [28]; we give here a new proof of this main result on mixed BC. If $\Gamma$ is at its degenerate scale for Dirichlet BC then there exists $q_{0} \neq 0$ such that:

$\int_{\Gamma} q_{0}(x) G(x, y) \mathrm{d} S(x)=0 \quad x \in \Gamma$.

We now consider the solution $u_{0}$ of the following auxiliary Laplace problem:

$\left\{\begin{array}{l}\Delta u_{0}=0 \quad x \in \Gamma^{+} \\ u_{0}(x)=0 \quad x \in \Gamma_{D} \\ \frac{\partial u_{0}}{\partial n}(x)=q_{0} \quad x \in \Gamma_{N} .\end{array}\right.$

The existence of this solution is a crucial point. For a connected in terior domain bounded by a finite number of simple curves of class $C^{2, \lambda}$ we can refer to [59].

This solution satisfies the following boundary integral equations:

$\begin{cases}\int_{\Gamma_{N}} u_{0}(y) H(x, y) \mathrm{d} S_{y}-\int_{\Gamma} \frac{\partial u_{0}}{\partial n} G(x, y) \mathrm{d} S_{y}=0 & x \in \Gamma_{D} \\ \frac{1}{2} u_{0}(x)+\int_{\Gamma_{N}} u_{0}(y) H(x, y) \mathrm{d} S_{y}-\int_{\Gamma} \frac{\partial u_{0}}{\partial n} G(x, y) \mathrm{d} S_{y}=0 & x \in \Gamma_{N} .\end{cases}$

Adding (8) to (10) we get the following equations:

$\begin{cases}\int_{\Gamma_{N}} u_{0}(y) H(x, y) \mathrm{d} S_{y}-\int_{\Gamma_{D}}\left(\frac{\partial u_{0}}{\partial n}-q_{0}\right) G(x, y) \mathrm{d} S_{y}=0 & x \in \Gamma_{D} ; \\ \frac{1}{2} u_{0}(x)+\int_{\Gamma_{N}} u_{0}(y) H(x, y) \mathrm{d} S_{y}-\int_{\Gamma_{D}}\left(\frac{\partial u_{0}}{\partial n}-q_{0}\right) G(x, y) \mathrm{d} S_{y}=0 & x \in \Gamma_{N} .\end{cases}$

So, we can conclude that $\left(u_{0}, \frac{\partial u_{0}}{\partial \eta}-q_{0}\right)$ is a solution of (7). If $q_{0}$ is non null on $\Gamma_{N}$, then $u_{0}$ is non null and then $u_{0}$ is non null on $\Gamma$. As $u_{0}$ is null on $\Gamma_{D}$, then we deduce that $u_{0}$ is non null on $\Gamma_{N}$ and then $\left(u_{0}, \frac{\partial u_{0}}{\partial n}-q_{0}\right)$ is non null on $\Gamma_{N} \times \Gamma_{D}$. If $q_{0}$ is null on $\Gamma_{N}$, we conclude that $\int_{\Gamma_{D}} q_{0} G(x, y) \mathrm{d} S_{y}=0$ with $q_{0}$ non null on $\Gamma_{D}$ and $\left(0, q_{0}\right)$ is a non null solution of (7), which proves that the domain is at a degenerate scale for mixed BC.

\subsection{Behavior if the mixed $B C$ problem is at degenerate scale}

The result of that section is that if the mixed $\mathrm{BC}$ problem is at degenerate scale then, the Dirichlet BC problem is also at degenerate scale. This result has been previously investigated in [19] using complex methods and we provide here a new proof of this fundamental property. We suppose now that there are non null functions $\left(u_{0}, q_{0}\right)$ defined on $\left(\Gamma_{N} \times \Gamma_{D}\right)$ satisfying (7). If we assume $u_{0}=0$, we define a function $q(x)$ by setting $q(x)=0$, if $x \in \Gamma_{N}$ and $q(x)=q_{0}(x)$, if $x \in \Gamma_{D}$. Substituting $u=0$ in (7), we conclude that $\int_{\Gamma} q(x) G(x, y) \mathrm{d} S_{y}=0$ and $\Gamma$ is at the degenerate scale for the Dirichlet BC.

We assume now that $u_{0} \neq 0$. And we consider the solution $u$ of the following auxiliary problem:

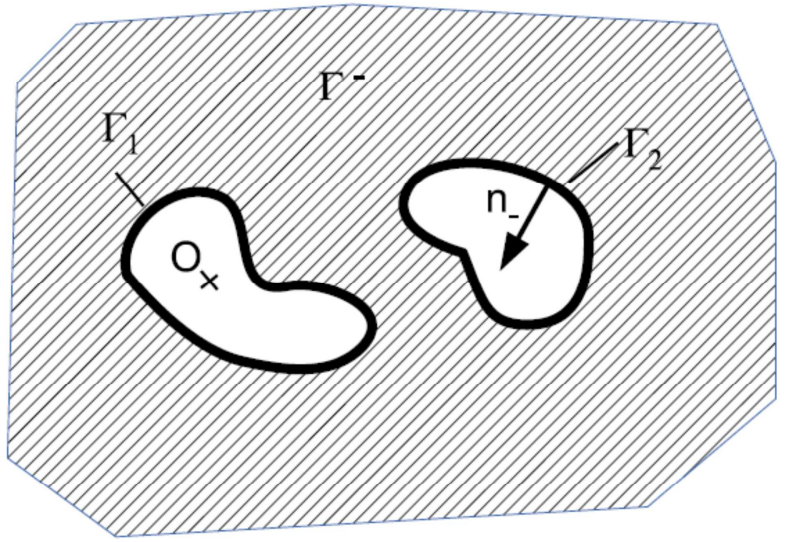

Fig. 1. Definition of $\Gamma^{-}, \Gamma=\Gamma_{1} \cup \Gamma_{2}$.

$\begin{cases}\Delta u=0 & x \in \Gamma^{+} \\ u(x)=0 & x \in \Gamma_{D} \\ u(x)=u_{0} & x \in \Gamma_{N} .\end{cases}$

Such a function satisfies the following boundary integral equations:

$\begin{cases}\int_{\Gamma_{N}} u(y) H(x, y) \mathrm{d} S_{y}-\int_{\Gamma} \frac{\partial u}{\partial n} G(x, y) \mathrm{d} S_{y}=0 & x \in \Gamma_{D} \\ \frac{1}{2} u(x)+\int_{\Gamma_{N}} u(y) H(x, y) \mathrm{d} S_{y}-\int_{\Gamma} \frac{\partial u}{\partial n} G(x, y) \mathrm{d} S_{y}=0 & x \in \Gamma_{N} .\end{cases}$

But $\left(u_{0}, q_{0}\right)$ is a solution of (7), and by subtracting (7) out of (13) we get:

$\begin{cases}\int_{\Gamma} \frac{\partial u}{\partial n}(y) G(x, y) \mathrm{d} S_{y}-\int_{\Gamma_{D}} q_{0}(y) G(x, y) \mathrm{d} S_{y}=0 & x \in \Gamma_{D} \\ \int_{\Gamma} \frac{\partial u}{\partial n}(y) G(x, y) \mathrm{d} S_{y}-\int_{\Gamma_{D}} q_{0}(y) G(x, y) \mathrm{d} S_{y}=0 & x \in \Gamma_{N} .\end{cases}$

We define $\tilde{q}$ by $\tilde{q}=\frac{\partial u}{\partial n}(x)$ for $x \in \Gamma_{N}$ and $\tilde{q}=\left(\frac{\partial u}{\partial n}(x)-q_{0}(x)\right)$ for $x \in \Gamma_{D}$, and we check that $\int_{\Gamma} \tilde{q}(y) G(x, y) \mathrm{d} S_{y}=0$. As $u \neq 0$ we must have $\frac{\partial u}{\partial n}(x) \neq$ 0 on $\Gamma_{N}$ and we conclude that $\tilde{q} \neq 0$. So the boundary $\Gamma$ is also at its degenerate scale in that case.

Finally, as there is one and only one degenerate scale for Dirichlet $\mathrm{BC}$, there is one and only one degenerate scale for the interior problem with mixed $\mathrm{BC}$.

\section{General results for an exterior Laplace problem with mixed BC}

The main result of that section is that an exterior Laplace problem with mixed BC has one and only one degenerate scale.

\subsection{Preliminaries}

Our results will rely on the existence and uniqueness of the solution of an exterior problem with mixed boundary conditions. Different cases have been studied, notably by Krutitskii $[59,60]$. We will refer here to the case of an exterior domain bounded by one or several simple closed curves of class $C^{2, \lambda}$ for $\lambda \in(0,1]$ [60] see Fig. 1 .

In the following, the finite set of the limit points where the type of the $\mathrm{BC}$ changes is denoted by $X$.

A function $u$ belongs to the smoothness class $K$ if:

$$
\left\{\begin{array}{l}
u \in C^{0}\left(\bar{\Gamma}^{-}\right) \cap C^{2}(\Gamma) ; \\
\nabla u \in C^{0}\left(\bar{\Gamma}^{-} \backslash X\right) ; \\
|\nabla u(y)|<c|x-y|^{\epsilon} \text { if }|x-y| \rightarrow 0, x \in X, c>0, \epsilon>-1 .
\end{array}\right.
$$


where $\bar{\Gamma}^{-}$is the closure of $\Gamma^{-}$(see Fig. 1) and $\bar{\Gamma}^{-} \backslash X$ is the set of points $x$ such that $x \in \bar{\Gamma}^{-}$and $x \notin X$.

We consider the following boundary problem:

$$
\left\{\begin{array}{l}
\Delta u(x)=0 x \in \Gamma^{-} ; \\
u(x)=f_{D}(x) x \in \Gamma_{D} ; \\
\frac{\partial u}{\partial n_{-}}(x)=f_{N}(x) ; x \in \Gamma_{N} .
\end{array}\right.
$$

The function defining the boundary conditions are assumed to be $C^{1, \lambda}$ for the parts with Dirichlet BC and $C^{0, \lambda}$ for the part with Neumann BC. We assume that the behavior at infinity assumes the following conditions:

$$
\left\{\begin{array}{l}
u(x)=\omega+\mathrm{O}\left(|x|^{-1}\right) \\
\nabla u(x)=\mathrm{O}\left(|x|^{-2}\right)
\end{array}\right.
$$

Then, if the measure of $\Gamma_{D}$ is not null, the problem defined by (16), (17) has a unique solution in $K$ [60]. (The condition on $u(x)$ is stated in [60] as $u(x)=\mathrm{O}(1)$ and $\nabla u(x)=\mathrm{o}\left(|x|^{-1}\right)$ but it is easily replaced by the more precise formulation (17)). The condition (17) is also used in [61]. This condition at infinity is not satisfying, because it can be fulfilled only if $\int_{\rho \Gamma} q \mathrm{~d} S_{y}=0$, i.e. if the the resultant of the flux on the inner boundary is null. However, for many practical problems, it is necessary to apply flux over the inner boundary whose resultant is not null (for example, in the case of normal flux constant over the boundary). So, a less restricting condition is introduced in Section 4.2, which allows to deal with a larger set of physical problems.

\subsection{Characterization of the degenerate scale with mixed $B C$}

We then consider the following problem : find $(u, \omega) \in K \times \mathbb{R}$ satisfying (16) with $f_{N}=0, f_{D}=0$ and the following condition at infinity:

$$
\left\{\begin{array}{l}
u(x)=-\frac{1}{2 \pi} \ln (|x|)+\omega+\mathrm{O}\left(|x|^{-1}\right) ; \\
\nabla u(x)=-\frac{1}{2 \pi} \nabla \ln (|x|)+\mathrm{O}\left(|x|^{-2}\right) .
\end{array}\right.
$$

This is the characterization of the degenerate scale used in [62] for Dirichlet condition. This condition accommodates now the case of a constant normal flux over the boundary. Then, we consider the following BC:

$$
\left\{\begin{array}{l}
f_{N}=-\frac{1}{2 \pi} \ln (|x|) x \in \Gamma_{D} \\
f_{D}=-\frac{1}{2 \pi} \frac{\partial \ln (|x|)}{\partial n_{-}} x \in \Gamma_{N} .
\end{array}\right.
$$

We denote by $v$ the unique solution of (16), (17), (19). It belongs to $K$. Then we consider $u(x)=-\frac{1}{2 \pi} \ln (|x|)-v(x)$. This is a solution $(u, \omega)$ of the problem defined by (20) and (17).

$$
\left\{\begin{array}{l}
\Delta u(x)=0 x \in \Gamma^{-} ; \\
u(x)=0 x \in \Gamma_{D} ; \\
\frac{\partial u}{\partial n_{-}}(x)=0 ; x \in \Gamma_{N} .
\end{array}\right.
$$

If we assume two solutions $\left(u_{1}, \omega_{1}\right),\left(u_{2}, \omega_{2}\right)$ of (20) and (18), then $u_{1}-u_{2}$ satisfies (16) and (17) with homogeneous conditions and hence is null and we get that $\omega_{1}=\omega_{2}$.

This allows to define the degenerate scale factor $\rho_{0}$ such that there is a function $u_{\rho}$ defined on the exterior of the homothetic contour $\rho_{0} \Gamma$ such that $u_{\rho}$ is the solution of (20) and (18) on $\rho_{0} \Gamma^{-}$with $\omega=0$. It can be seen easily that the choice $u_{\rho}(x)=u(x / \rho)$ and $\rho=e^{-2 \pi \omega}$ satisfies these conditions.

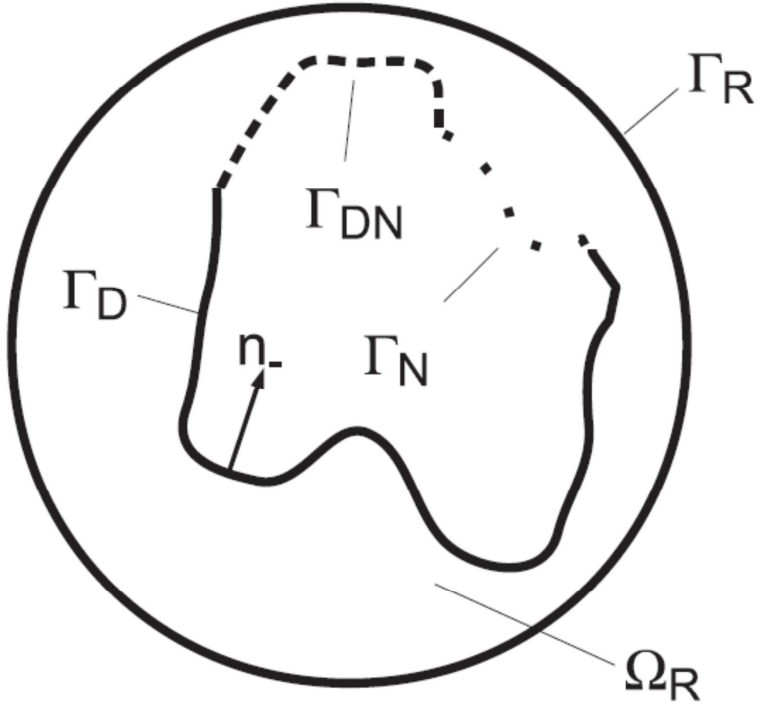

Fig. 2. Definition of $\Gamma_{D}, \Gamma_{N}, \Gamma_{D N}, \Gamma_{R}, \Omega_{R}$.

4.3. Behavior of the degenerate scale factor when the part of the contour submitted to Neumann BC increases

The main result of this section is that the degenerate scale factor is an increasing function of the part of the contour submitted to Neumann BC.

We will compare the degenerate scale for two decompositions of the contour and denote $u_{1}$ and $u_{2}$ the solutions related to these two kinds of BC. We assume that for $u_{2}$ the part of the contour submitted to Neumann condition $\Gamma_{N 2}$ is larger than for $u_{1}$; more precisely, we assume that: $\Gamma_{N 1} \subset \Gamma_{N 2}$. We write: $\Gamma=\Gamma_{D} \cup \Gamma_{N} \cup \Gamma_{D N}$, with $\Gamma_{D}=\Gamma_{D_{1}} \cap \Gamma_{D_{2}}=\Gamma_{D_{2}}$, $\Gamma_{N}=\Gamma_{N_{1}} \cap \Gamma_{N_{2}}=\Gamma_{N_{1}} ; \Gamma_{D N}=\Gamma_{D 1} \cap \Gamma_{N 2}$ (see Fig. 2).

We write the Green's first identity for the function $\left(u_{2}-u_{1}\right)$ on the domain $\Omega_{R}$ :

$$
\begin{aligned}
& \int_{\Omega_{R}}\left(u_{2}-u_{1}\right) \Delta\left(u_{2}-u_{1}\right) \mathrm{d} V_{y}=0 \\
= & \underbrace{\int_{\Gamma}\left(u_{2}-u_{1}\right) \frac{\partial\left(u_{2}-u_{1}\right)}{\partial n_{-}} \mathrm{d} S_{y}}_{I}+\underbrace{\int_{\Gamma_{R}}\left(u_{2}-u_{1}\right) \frac{\partial\left(u_{2}-u_{1}\right)}{\partial r} \mathrm{~d} S_{y}}_{I_{R}} \\
& -\underbrace{\int_{\Omega_{R}}\left(\nabla\left(u_{2}-u_{1}\right)\right)^{2} \mathrm{~d} V_{y}}_{I_{\Omega_{R}} \geq 0} .
\end{aligned}
$$

The term $I_{\Omega_{R}}$ is actually strictly positive if $\Gamma_{N_{2}} \backslash \Gamma_{N 1}$ has a non null length. If $I_{\Omega_{R}}=0$, then, $u_{2}-u_{1}=$ Const. This constant is null because we assume that $\Gamma_{D}$ contains an open portion of the boundary with a non null length. Then $u_{2}=u_{1}$ is a harmonic function which is such that $u=\frac{\partial u}{\partial n}=0$ on a smooth open portion of the boundary. As a consequence, $u=0$, from [63]. As $u_{1} \neq 0, u_{2} \neq 0$, we conclude that $I_{\Omega_{R}}>0$.

Using the boundary conditions we get:

$$
\begin{aligned}
I= & \int_{\Gamma_{D}}\left(u_{2}-u_{1}\right) \frac{\partial\left(u_{2}-u_{1}\right)}{\partial n_{-}} \mathrm{d} S_{y}+\int_{\Gamma_{N}}\left(u_{2}-u_{1}\right) \frac{\partial\left(u_{2}-u_{1}\right)}{\partial n_{-}} \mathrm{d} S_{y} \\
& +\int_{\Gamma_{D N}}\left(u_{2}-u_{1}\right) \frac{\partial\left(u_{2}-u_{1}\right)}{\partial n_{-}} \mathrm{d} S_{y} \\
= & -\int_{\Gamma_{D N}} u_{2} \frac{\partial u_{1}}{\partial n_{-}} \mathrm{d} S_{y} .
\end{aligned}
$$




$$
\begin{aligned}
I_{R} & =\int_{\Gamma_{R}}\left(u_{2}-u_{1}\right) \frac{\partial\left(u_{2}-u_{1}\right)}{\partial r} \mathrm{~d} S_{y} \\
& =\int_{0}^{2 \pi}\left(\omega_{2}-\omega_{1}+\mathrm{O}\left(\frac{1}{R}\right)\right)\left(-\frac{1}{2 \pi R}+\frac{1}{2 \pi R}+\mathrm{O}\left(\frac{1}{R^{2}}\right)\right) R \mathrm{~d} \theta=\mathrm{O}\left(\frac{1}{R}\right) .
\end{aligned}
$$

Using (21)-(23), we get:

$$
I=-\int_{\Gamma_{D N}} u_{2} \frac{\partial u_{1}}{\partial n_{-}} \mathrm{d} S_{y}>0 .
$$

We now write the Green's second identity for the functions $\left(u_{2}, u_{1}\right)$ on the domain $\Omega_{R}$ :

$$
\begin{aligned}
& \int_{\Omega_{R}}\left(u_{2} \Delta u_{1}-u_{1} \Delta u_{2}\right) \mathrm{d} V_{y}=0 \\
= & \underbrace{\int_{\Gamma}\left(u_{2} \frac{\partial u_{1}}{\partial n_{-}}-u_{1} \frac{\partial u_{2}}{\partial n_{-}}\right) \mathrm{d} S_{y}}_{I^{\prime}}+\underbrace{\int_{\Gamma_{R}}\left(u_{2} \frac{\partial u_{1}}{\partial r}-u_{1} \frac{\partial u_{2}}{\partial r}\right) \mathrm{d} S_{y}}_{I_{R}^{\prime}} .
\end{aligned}
$$

It can been seen easily that $I^{\prime}=-I$. Let us evaluate now $I_{R}^{\prime}$ :

$$
\begin{aligned}
I_{R}^{\prime}= & \int_{\Gamma_{R}}\left(u_{2} \frac{\partial u_{1}}{\partial r}-u_{1} \frac{\partial u_{2}}{\partial r}\right) \mathrm{d} S_{y} \\
= & \int_{0}^{2 \pi}\left[\left(-\frac{1}{2 \pi} \ln (R)+\omega_{2}+\mathrm{O}\left(\frac{1}{R}\right)\right)\left(-\frac{1}{2 \pi R}+\mathrm{O}\left(\frac{1}{R^{2}}\right)\right)\right. \\
& \left.-\left(-\frac{1}{2 \pi} \ln (R)+\omega_{1}+\mathrm{O}\left(\frac{1}{R}\right)\right)\left(-\frac{1}{2 \pi R}+\mathrm{O}\left(\frac{1}{r^{2}}\right)\right)\right] R \mathrm{~d} \theta \\
= & \int_{0}^{2 \pi}\left(\frac{1}{2 \pi}\left(\omega_{1}-\omega_{2}\right)+\mathrm{O}\left(\frac{\ln (R)}{R}\right)\right) \mathrm{d} \theta .
\end{aligned}
$$

When $R \rightarrow \infty, I_{R}^{\prime} \rightarrow\left(\omega_{1}-\omega_{2}\right)$. Combining (24)-(26), we get:

$$
I=-I^{\prime}=I_{R}^{\prime}=\omega_{1}-\omega_{2}>0 \text {. }
$$

We finally conclude:

$\rho_{2}=e^{-2 \pi \omega 2}>e^{-2 \pi \omega 1}=\rho_{1}$,

which proves that the degenerate scale factor is a growing function of the part of the contour submitted to Neumann BC.

\section{Degenerate scale of a segment for mixed BC}

5.1. Comparison of the degenerate scale of a segment submitted to mixed $B C$ and the degenerate scale of the part of the segment to Dirichlet $B C$

The main result of this section is that the degenerate scale for a segment with mixed $\mathrm{BC}$ is equal to the degenerate scale of the part of the segment subjected to Dirichlet condition.

We consider a segment $S$. It is split into a finite number of segments $S_{D_{i}}$ and $S_{N_{i}}$ with respectively Dirichlet and Neumann conditions (the same condition on both sides). If the set of segments $\left(\cup S_{D_{i}}\right)$ is at its degenerate scale for Dirichlet $\mathrm{BC}$, there exists a non null solution $u$ to the problem (29).

$\left\{\begin{array}{l}\Delta u=0 \quad x \in\left(\cup S_{D_{i}}\right)^{-} ; \\ u(x)=0 \quad x \in \cup S_{D_{i}} ; \\ u(x)=-\frac{1}{2 \pi} \ln |x|+\mathrm{o}\left(|x|^{-1}\right) \quad|x| \rightarrow \infty .\end{array}\right.$

Due to the symmetry, the derivative $\frac{\partial u}{\partial n}=0$ for $x$ in the interior of $\cup S_{N_{i}}$. We conclude that the segment $S$ is at the degenerate scale for the mixed BC.
5.2. Some exact values

It is possible to find exact values for some sets of aligned segments by the "pullback formula" (see for example [55]). In the same reference, the solution for two equal segments with Dirichlet $\mathrm{BC}[-a,-b] \cup[b, a]$ (with $a>b>0$ ) is given: the set is at the degenerate scale if

$\sqrt{a^{2}-b^{2}}=2$,

and some other examples with 3 segments are also given. An example with 4 segments can been found in [64]. This paper provides also the literature on the methodology leading to these results, that falls outside the scope of our present work.

In this special case, the fact that the degenerate scale factor is a growing function of the part submitted to Neumann BC is a direct consequence of the fact that the logarithmic capacity is a growing function of the considered domain.

\section{Degenerate scale for an exterior problem of a circle with mixed} BC

6.1. Relation between mixed boundary value problem on a circle and a similar problem on a contour obtained by conformal mapping

We suppose that there is a conformal mapping $w$ from the outside of a circle $C$ to the outside of the considered simple curve $\Gamma$ such that $w(z)=z+\mathrm{O}(1 / z)$ when $|z| \rightarrow \infty$. If $u(z)$ is a solution of a mixed problem on the circle, then $\Upsilon(\zeta)=u\left(w^{-1}(\zeta)\right)$ is a solution of a mixed BC problem on $\Gamma$ where the part of $\Gamma$ submitted to Dirichlet $B C$ is the image of the part of $C$ submitted to Dirichlet BC. The boundary is an equipotential line for the part of it with Dirichlet condition and a streamline for the part of it with Neumann BC. The image of the stream lines and of the equipotential lines of $u$ are the stream lines and the equipotential lines of $Y$ (see e.g. [65]). And so $Y$ is a harmonic function which satisfies the mixed BC. The last point to check is the radiation condition and, from the definition of the conformal mapping, it can be seen easily that $Y(\zeta)$ behaves at $\infty$ like $u(z)$.

We can conclude finally that if a circle $C$ with Dirichlet $\mathrm{BC}$ on $C_{D}$ and Neumann BC on $C_{N}$ is at the degenerate scale for the exterior Laplace problem then, $\Gamma=w(C)$ with Dirichlet BC on $w\left(C_{D}\right)$ and Neumann BC on $w\left(C_{N}\right)$ is also at the degenerate scale.

\subsection{Conformal mapping of a circle onto a segment}

It is well known that $w(z)=z+R^{2} / z$ maps the circle with radius $\mathrm{R}$ and center at the origin, onto the segment $[-2 R, 2 R]$. We use the previous results on conformal mapping for the segment. For that, it is important that both sides of the segment (upper and bottom side) have the same kind of $\mathrm{BC}$ as we use a symmetry argument along the real axis. So we restrict ourselves to the case where the part $C_{D}$ (and consequently the part $C_{N}$ ) is symmetric with respect to the real axis.

Then the circle is at the degenerate scale if and only if the set of segments $w\left(C_{D}\right)$ is at the degenerate scale. We give now some examples.

\subsection{Case when $\mathrm{C}_{\mathrm{D}}$ is connected}

We consider a circle with radius $R$ and the part $C_{D}$ with Dirichlet BC is connected with length $2 R \alpha$.

The circle is at its degenerate scale if the image of its part submitted to Dirichlet BC which is the segment of length $2 R(1-\cos (\alpha))$ is at its degenerate scale, that is if its length is equal to 4 . We get the condition $2 R(1-\cos (\alpha))=4$ which can be written, using $(1-\cos (\alpha))=2 \sin ^{2}(\alpha / 2)$, in the following way:

$R \sin ^{2}(\alpha / 2)=1$.

This condition is different from the condition for $C_{D}$ to be at the degenerate scale: this condition writes $R \sin (\alpha / 2)=1$ [17]: the useful property, 


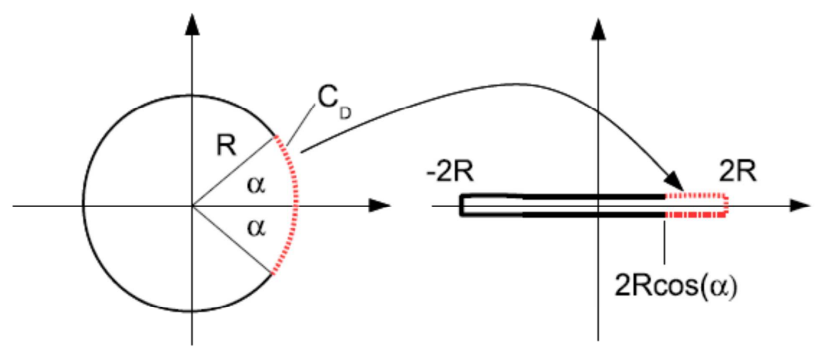

Fig. 3. Case of a circle with one part with Dirichlet condition.

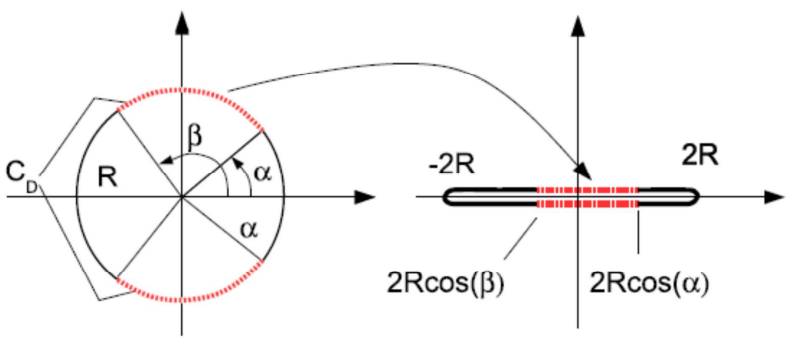

Fig. 4. Case of a circle with two parts with Dirichlet condition.

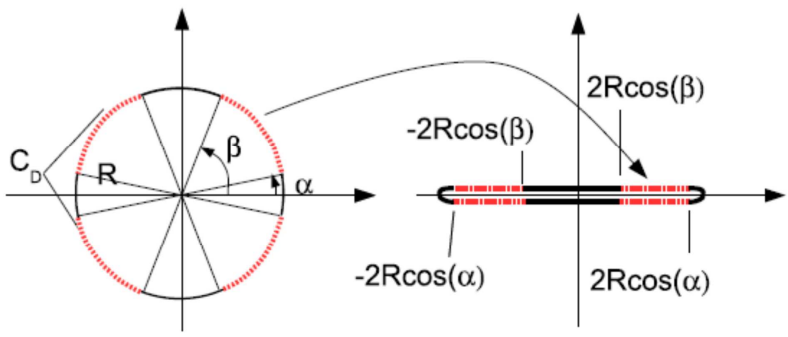

Fig. 5. Case of a circle having four parts with Dirichlet condition.

relating the degenerate scale for mixed $\mathrm{BC}$ to the degenerate scale of the part at Dirichlet BC, is true for a segment, but cannot be extended to any boundary curve, as shown in the case of the circle. The condition giving the degenerate scale for mixed BC with Dirichlet BC on the half circle, i.e. $\alpha=\pi / 2$ is $R=2$.

\subsection{Case when $\mathrm{C}_{\mathrm{D}}$ has two connected parts}

We consider the case with $C_{D}$ constituted by two symmetric arcs of angle $(\beta-\alpha)$ (see Fig. 4).

The condition for $C_{D}$ being at the degenerate scale is: $2 R(\cos (\alpha)-$ $\cos (\beta))=4$. We find the value of the degenerate scale factor $R$ :

$$
R=\frac{1}{\sin \left(\frac{\beta-\alpha}{2}\right) \sin \left(\frac{\alpha+\beta}{2}\right)} \text {. }
$$

A special symmetric case is $\alpha=\pi / 4 ; \beta=3 \pi / 4$. Then, the total length of $C_{D}$ is $\pi R$ and the degenerate scale factor is:

$$
R=\sqrt{2} \text {. }
$$

It is smaller than for one arc having the same length.

\subsection{Case with four connected parts with Dirichlet $B C$}

We consider now a set of four arcs symmetric with respect to the real and the imaginary axes (Fig. 5).

Using (30), we get the following condition:

$R \sqrt{\cos ^{2}(\alpha)-\cos ^{2}(\beta)}=R \sqrt{\sin (\beta-\alpha) \sin (\alpha+\beta)}=1$.
As an example, if $\alpha=\pi / 8$ and $\beta=3 \pi / 8$, the total length of $C_{D}$ is $\pi R$ and the condition is:

$R=\sqrt[4]{2}$.

6.6. Case of a circle where the part submitted to the Dirichlet BC has a n-fold symmetry

Then, we can evaluate the degenerate scale of a n-fold symmetric problem on the unit circle with a n-fold symmetric part submitted to Dirichlet $\mathrm{BC} C_{n, D}$, if we know the degenerate scale of the problem on the unit circle with the Dirichlet $\mathrm{BC}$ on $C_{D}$ such that, using polar coordinates, $\left((1, \theta) \in C_{n, D} \Leftrightarrow(1, n \theta) \in C_{D}\right)$.

We consider the function $u$ solution of (18), (20) of the problem with $C_{D}$ and we define $u_{n}(r, \theta)=\frac{1}{n} u\left(r^{n}, n \theta\right)$. It can be seen easily that $u$ is a harmonic function outside the unit circle. It also satisfies the following BC: $u_{n}=0$ on $C_{n, D}, \partial u_{n} / \partial \theta=0$ on $C_{n, N}$. Checking the radiative condition, we see that when $r \rightarrow \infty$ :

$$
\begin{aligned}
& u_{n}(r, \theta)=\frac{1}{n} u\left(r^{n}, n \theta\right) \\
= & -\frac{1}{n} \frac{1}{2 \pi} \ln \left(r^{n}\right)+\frac{\omega}{n}+\mathrm{O}\left(\frac{1}{r}\right)=-\frac{1}{2 \pi} \ln (r)+\frac{\omega}{n}+\mathrm{O}\left(\frac{1}{r}\right) .
\end{aligned}
$$

So, we deduce that the degenerate scale factor $R_{n}$ of the $n$-fold symmetric problem is $R^{\frac{1}{n}}$. The preceding results (33), (35), appear to be particular cases.

\section{Degenerate scales with mixed BC for contours having a line symmetry}

\subsection{Case when the Dirichlet $B C$ is applied to one half of the boundary}

We consider a simple contour with a symmetry axis and the following mixed BC: the Dirichlet BC is applied to one of the two symmetric parts, the Neumann BC is applied to the other one (Fig. 6).

The half part is mapped on the half part of the unit circle which is mapped on the half of the segment of length 4 . We deduce that the degenerate scale factor for this particular mixed $\mathrm{BC}$ is twice the degenerate scale factor for Dirichlet BC.

7.2. Case when the part submitted to Dirichlet BC has the same symmetry line as the boundary

In that case, it is also possible to reduce the problem of finding the degenerate scale of the mixed $\mathrm{BC}$ problem on $\Gamma$ to the evaluation of the logarithmic capacity of a set of aligned segments (Fig. 7).

\section{Case of figures with a n-fold symmetry}

8.1. Case when $\Gamma_{\mathrm{D}}$ is a connected part equal to the fraction $\mathrm{k} / \mathrm{n}$ of the whole boundary

We suppose that a connected part corresponding to $1 / n$ of the whole curve is submitted to Dirichlet BC (Fig. 8). The conformal mapping maps this part to an arc of circle of angle $2 \pi / n$. Using the result of Section 6.1 and Eq. (31), we deduce that the degenerate scale factor is

$R=\frac{1}{\sin ^{2}(\pi / 2 n)}$

if the curve is at its degenerate scale for Dirichlet BC.

If the part submitted to Dirichlet $\mathrm{BC}$ is the fraction $k / n$ connected part of the whole curve, we get :

$R=\frac{1}{\sin ^{2}(\pi k / 2 n)}$. 


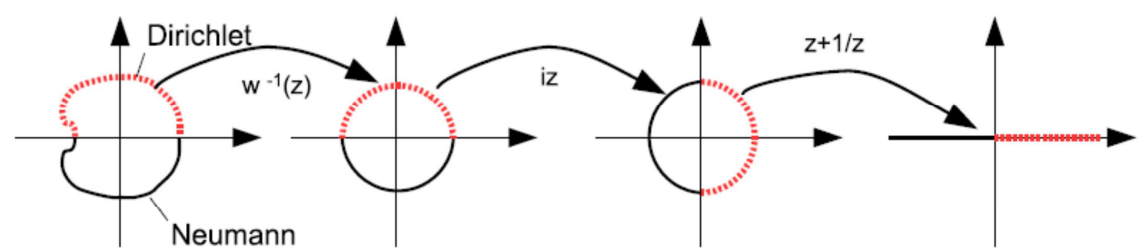

Fig. 6. Case of a figure with a symmetry axis, the part submitted to Dirichlet BC being symmetric of the other part.

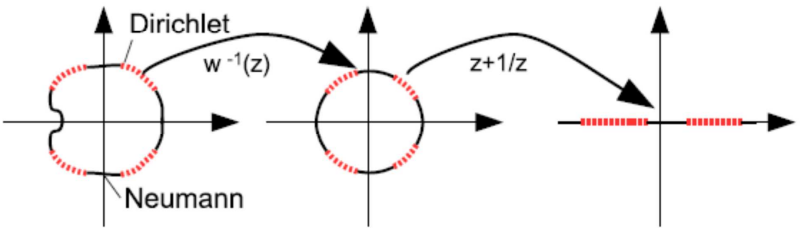

Fig. 7. Case of a figure with a symmetry axis, the part submitted to Dirichlet $\mathrm{BC}$ being symmetric with respect to the symmetry axis.

\subsection{Case when $\Gamma_{\mathrm{D}}$ is a set of $n$ arcs with the $n$-fold symmetry}

We denote by $\beta$ the arc of circle corresponding by the conformal mapping to the $1 / n$ part submitted to the Dirichlet BC (Fig. 9). Then the angle in the image circle by $z^{n}$ is $n \beta$ and applying (31) and (36) we get:

$R=\left(\frac{1}{\sin ^{2}\left(\frac{n \hat{\rho}}{4}\right)}\right)^{\frac{1}{n}}$

It is interesting to notice that if the part submitted to Dirichlet $\mathrm{BC}$ is constant, $n \beta=$ Const $<2 \pi$, then when $n \rightarrow \infty, R \rightarrow 1$ : when the part submitted to Dirichlet $\mathrm{BC}$ becomes more and more finely divided, the degenerate scale factor tends to the degenerate scale factor for the Dirichlet case.

\section{Applications}

\subsection{Case of an equilateral triangle}

The use of conformal mapping allows to compare this case with the case of the circle.

Thanks to the symmetry, it is easy to find the points of the circle corresponding by the Schwarz-Christoffel mapping to the vertices and to the middles of the sides. The results are given in Table 1 for 11 cases of mixed BC.

In that table, we find the ratio $\rho_{m} / \rho_{D}$. $\rho_{D}$ is the degenerate scale factor that transforms the equilateral triangle (with Dirichlet boundary condition) with $h$ height to the degenerate scale triangle with height $h_{D}=$ $\rho_{D} h \approx 2.053$ (and Dirichlet boundary condition). $\rho_{m}$ is the degenerate scale factor that transforms the equilateral triangle (with mixed boundary condition) with $h$ height to the degenerate scale triangle for mixed boundary condition, having height $h_{m}=\rho_{m} h$. The ratio $\rho_{m} / \rho_{D}=h_{m} / h_{d}$ does not depend on the original scale of the triangle.
The cases (1-9) can be solved by using (31). The case (7) is a direct application of Section 7.1. The solutions of cases (11-12) are found by using the results of Section 7.2 and Eq (32). The case (10) needs to consider the inverse image of the triangle by the Schwarz-Christoffel transform and to use Eq. (32) after a rotation. The case (13) is solved with the help of Section 8.2 and Eq. (39).

As an example, let us detail the case 2 of Table 1. Due to the symmetry, the half side is mapped by the inverse of the Schwarz-Christoffel mapping to the $1 / 6$ part of the circle. Then, the angle $\alpha$ in Fig. 3 is equal to $\pi / 6$. Applying (31), the condition is $R=\rho_{m} / \rho_{D}=1 / \sin ^{2}(\alpha / 2)=$ $1 / \sin ^{2}(\pi / 12)$. We have $\pi / 12=\sqrt{2}(\sqrt{3}-1) / 4$ see for e.g. [66]. An easy calculus then results in $R=4(2+\sqrt{3})$.

Looking at cases $(1,2,4,5,7,8,9)$ allows to verify that the degenerate scale factor decreases when the part of the boundary submitted to Dirichlet BC increases. We conclude in the same way when considering cases $(1,2,10,7,9)$ and $(1,2,10,13,9)$. When comparing cases $(4,10$, $11)$, it can been seen that the degenerate scale factor is smaller when the distance between two half sides submitted to the Dirichlet BC is larger.

\subsection{Case of a square}

The results for different configurations of the mixed $\mathrm{BC}$ are given in Table 2 for 16 cases of mixed BC. The method is the same as for the equilateral triangle: cases (1-10) need (31), case (6) is also solved by using Section 7.1. Cases $(13,15,16)$ can be found using the $n$-fold symmetry (Section 8.2). Cases $(12,14)$ can be solved using Section 7.2 . Case (11) needs a direct application of (32) on the inverse image of the problem by the Schwarz-Christoffel mapping.

As an example, let us detail the case 12 of Table 2. Due to the symmetry, the half side is mapped by the inverse of the SchwarzChristoffel mapping to the $1 / 8$ part of the circle. Then, the angle $\alpha$ in Fig. 4 is equal to $\pi / 4$ and $\beta=\pi / 2$. Applying (32), the condition is $R=1 /(\sin (\pi / 8) \sin (3 \pi / 8))$. We have $\sin (\pi / 8)=\sqrt{(1-\sqrt{2} / 2) / 2}$ and $\sin (3 \pi / 8)=\sqrt{(1+\sqrt{2} / 2) / 2}$ see for e.g. [66]. An easy calculus then results in $R=2 \sqrt{2}$ ).

When comparing the cases $(1,2,4,5,6,7,8,9,10)$, it can be seen again that when $\Gamma_{D}$ increases, the degenerate scale factor decreases. When comparing the cases $(3,4,11,12,13,14)$ or the cases $(6,15$, 16 ), we notice that the more homogeneous is the repartition of $\Gamma_{D}$, the smaller is the degenerate scale factor.

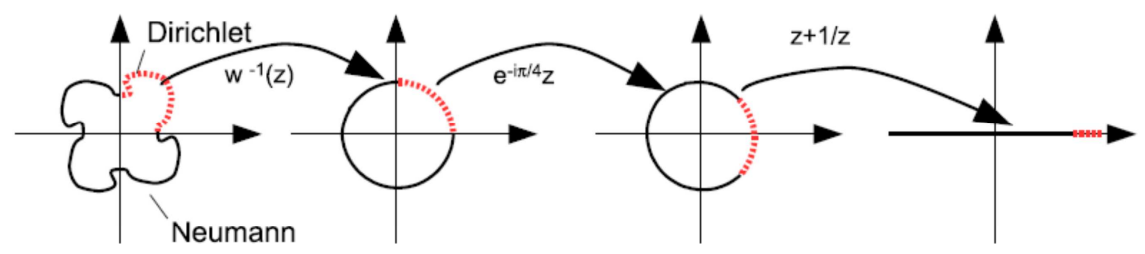

Fig. 8. Case of a figure with a $n$-fold symmetry, the part submitted to Dirichlet BC being the fraction $k / n$ of the whole boundary. 


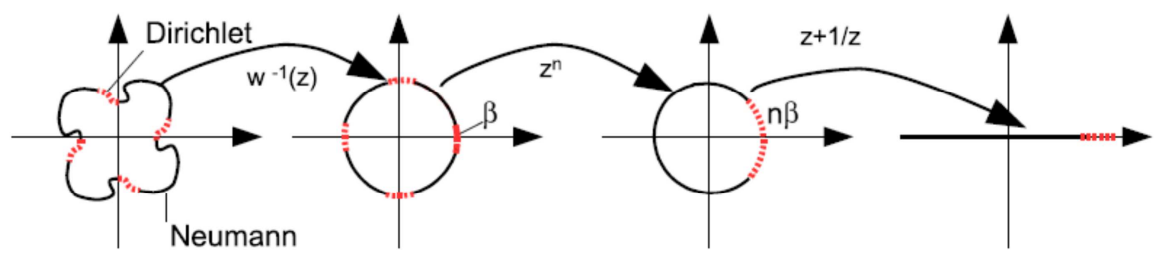

Fig. 9. Case of a figure with a $n$-fold symmetry, the part submitted to Dirichlet $\mathrm{BC}$ having the same symmetry.

Table 1

Value of the ratio for $\frac{\rho_{m}}{\rho_{D}}$ for different mixed boundary conditions on an equilateral triangle. $\left(\rho_{m}\left(\rho_{D}\right)\right.$ is the degenerate scale factor for mixed $\mathrm{BC}$ (respectively Dirichlet $\mathrm{BC}$ ), $\rho_{\mathrm{D}} \approx 2.053 / \mathrm{h}$ with $\mathrm{h}$ the height of the equilateral triangle).

Case Figure

10. Numerical determination of the degenerate scale factor for mixed BC

In that section, we consider the exterior problem.

\subsection{Method of numerical determination of the degenerate scale}

The numerical determination of the degenerate scale factor rests on the boundary element formulation of the integral equation. It will use standard direct formulation of the BEM and the mathematical tool of generalized eigenvalue as already used in [41] for the case of Robin BVP and described in [52] for the case of Laplace equation in the half-space.

We use the singular BIE and not the null field BIE as in $[67,68]$.

The boundary element formulation is written by using the Green's function of Laplace equation defined up to a constant $C$ by:

$G=-\frac{1}{2 \pi} \ln (r)$,
Let us consider the problem related to the boundary conditions $u=u_{0}$ over $\Gamma_{D}$ and $\frac{\partial u}{\partial n}=q_{0}$ over $\Gamma_{N}$. The boundary element numerical representation of the integral equation is formulated in this work by using constant elements and can be written as:

$\left[H_{D}, H_{N}\right]\left[\begin{array}{l}u_{D} \\ u_{N}\end{array}\right]=\left[G_{D}, G_{N}\right]\left[\begin{array}{l}q_{D} \\ q_{N}\end{array}\right]$

with the obvious notation that index $D(N)$ refers to the nodes where Dirichlet (Neumann) BC is applied. $H_{D}$ and $G_{D}$ are rectangular matrices obtained by keeping the columns related to nodes on boundary at Dirichlet BC and identically for $H_{N}$ and $G_{N}$

This can be written by putting into the right hand side the known quantities as:

$\left[-G_{D}, H_{N}\right]\left[\begin{array}{c}q_{D} \\ u_{N}\end{array}\right]=\left[-H_{D}, G_{N}\right]\left[\begin{array}{l}u_{D} \\ q_{N}\end{array}\right]$.

the unknown being obviously $u_{N}, q_{D}$. 
Table 2

Value of the ratio $\frac{\rho_{m}}{\rho_{0}}$ for different mixed boundary conditions on a square. $\left(\rho_{m}\left(\rho_{D}\right)\right.$ is the degenerate scale factor for mixed BC (respectively Dirichlet BC), $\rho_{\mathrm{D}} \approx 1.699 / \mathrm{a}$, with a the side of the square).

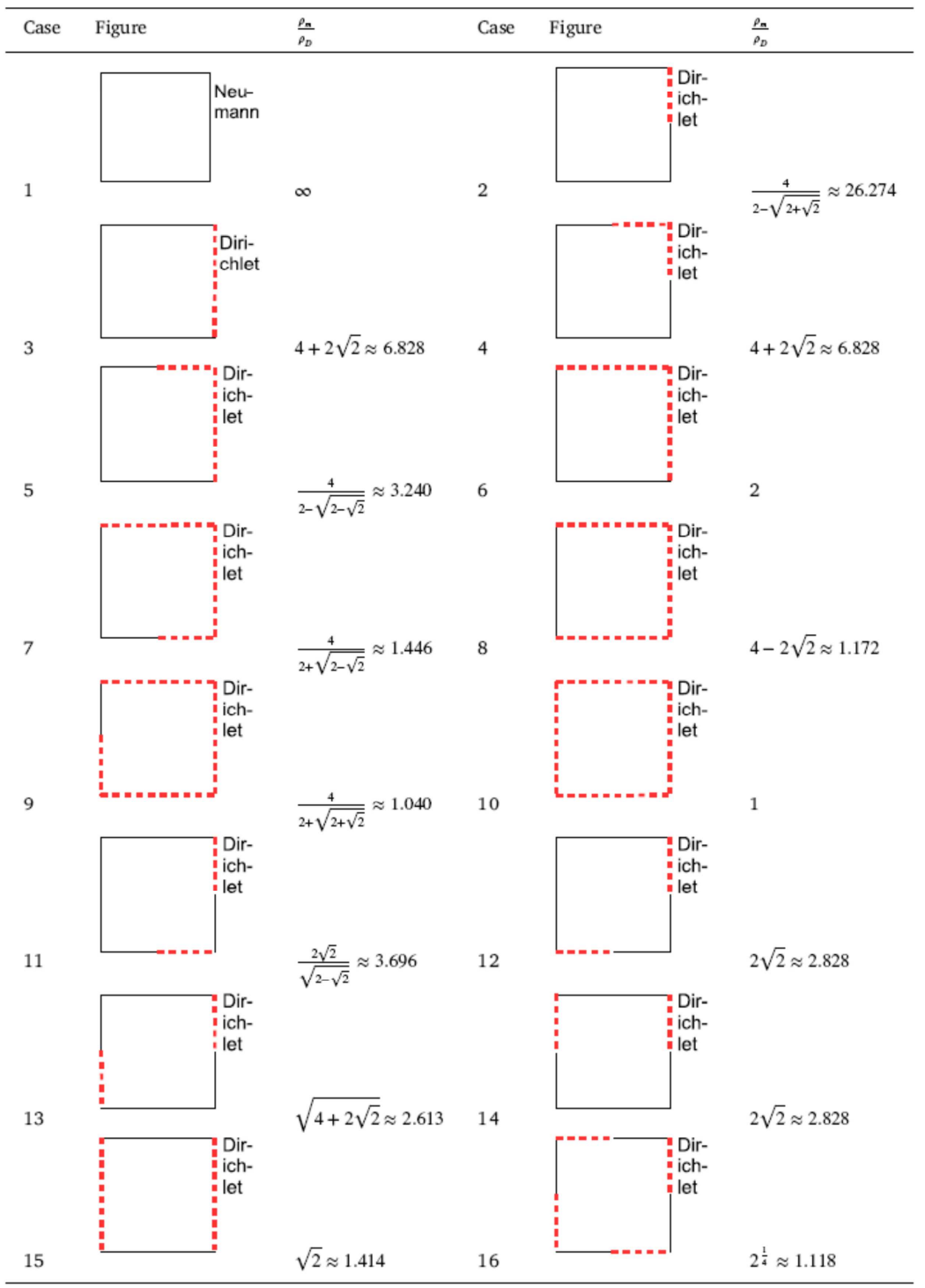

The scale factor is introduced by using the matrix $\left[G_{\rho}\right]$ related to $\rho \Gamma$ as a function of $[G]$ built from $\Gamma$. It is shown in Appendix A that the relation between these two matrices is:

$\left[G_{\rho}\right]=[G]+C[B]$,

where the computation of $[B]$ is described in Appendix A and with $C=$ $-\frac{1}{2 \pi} \ln (\rho)$. Introducing this into (42), the numerical formulation of the mixed BIE for the domain with boundary $\rho \Gamma$ becomes:

$\left[-G_{D}-C B_{D}, H_{N}\right]\left[\begin{array}{l}q_{D} \\ u_{N}\end{array}\right]=\left[-H_{D}, G_{N}+C B_{N}\right]\left[\begin{array}{l}u_{D} \\ q_{N}\end{array}\right]$.

If the domain with boundary $\rho \Gamma$ is at the degenerate scale, it means that matrix $\left[-G_{0 D}-C B_{D}, H_{N}\right]$ is singular, i.e. the system

$\left[-G_{0 D}-C B_{D}, H_{N}\right][X]=0$

has a non null solution. This can be written as:

$\left[-G_{0 D}, H_{N}\right][X]=C\left[B_{D}, 0\right][X]$.

Therefore, $C$ is a generalized eigenvalue of $\left(\left[-G_{0 D}, H_{N}\right],\left[B_{D}, 0\right]\right)$. This generalized eigenvalue can be computed by using standard nu- merical packages, for example in Matlab software. Looking for these generalized eigenvalues, it is found that most of these eigenvalues are very large (near the limit for digitized numbers). Therefore, it is better to look for the eigenvalues $\mu_{n}$ of $\left(\left[B_{D}, 0\right],\left[-G_{D}, H_{N}\right]\right)$. It is found that only one eigenvalue $\mu$ is not (nearly) null and finally $C$ is given by:

$C=\frac{1}{\mu}=\frac{1}{2 \pi} \log (a)=-\frac{1}{2 \pi} \log (\rho)$.

The degenerate scale factor is finally given by:

$\rho=e^{-2 \pi / \mu}$.

\subsection{Applications}

The numerical method for obtaining the degenerate scale factor has been applied to a circle of radius 1 . Fig. 10 shows the comparison between the analytical solution obtained in the previous section and the numerical one for a circle with an increasing part of the contour concerned by a Dirichlet BC, while on the remaining part a Neumann BC is applied. The numerical results have been obtained by using BEM with 


\begin{tabular}{lllll}
\hline Case & Dirichlet $\mathrm{BC}$ & Robin $\mathrm{BC}[53]$ & mixed $\mathrm{BC}$ & Neumann $\mathrm{BC}$ \\
\hline Interior problem & One degenerate scale $\rho_{D}$ & One degenerate scale equal to $\rho_{D}$ & $\begin{array}{l}\text { One degenerate scale factor equal to } \\
\rho_{D}[19][28]\end{array}$ & $\begin{array}{l}\text { No degenerate scale; one solution up } \\
\text { to a constant if the prescribed flux } \\
f_{\Gamma} q=0\end{array}$ \\
Exterior problem & $\begin{array}{l}\text { One degenerate scale factor } \rho_{D}, \text { the } \\
\text { same as for the interior problem }\end{array}$ & $\begin{array}{l}\text { One degenerate scale factor } \rho_{R} \geq \rho_{D} \\
\rho_{R} \text { increases when the parameter } t \text { in } \\
\text { the Robin BC decreases } \frac{\partial u}{d n}-t u=0\end{array}$ & $\begin{array}{l}\text { One degenerate scale factor } \rho_{m}>\rho_{D} \\
\rho_{m} \text { increases when the part subjected } \\
\text { to Neumann condition increases }\end{array}$ & $\begin{array}{l}\text { to a constant } \\
\text { theale; one solution up }\end{array}$ \\
\hline
\end{tabular}

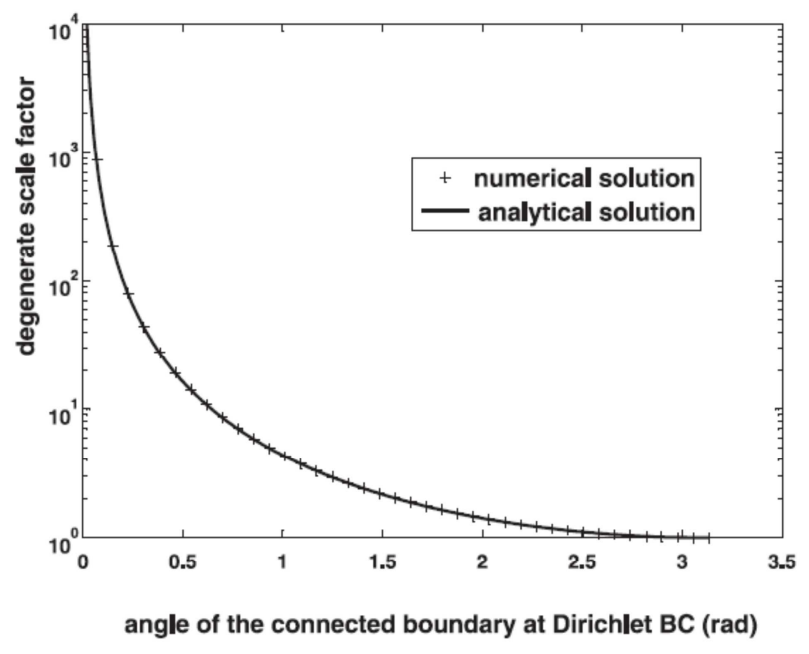

Fig. 10. Comparison between analytical and numerical values of the degenerate scale factor for a circle with increasing Dirichlet BC.

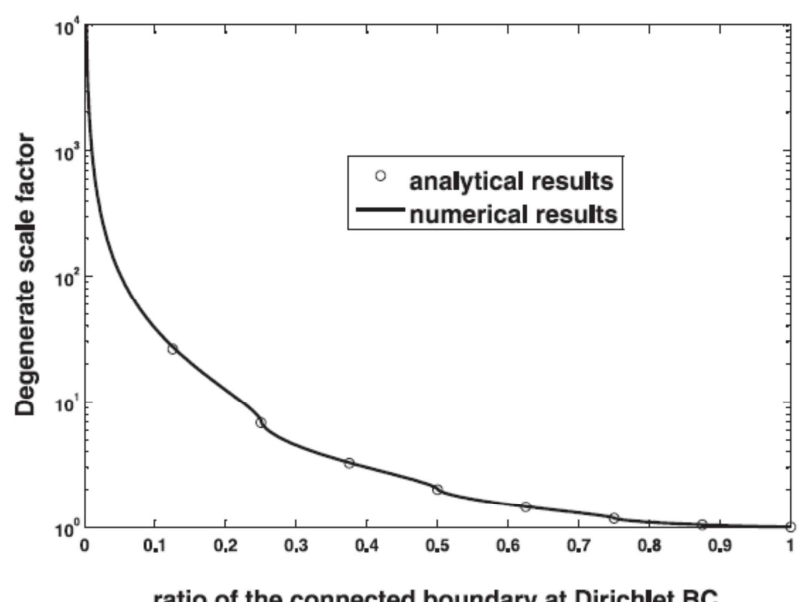

Fig. 11. Comparison between analytical and numerical values of the degenerate scale factor for a square with increasing Dirichlet BC, starting from one vertex.

400 constant elements. The results show that both analytical and numerical methods produce the same results over all the range of the increasing boundary. Fig. 11 produces similar results for the case of a square which is at the degenerate scale for Dirichlet BC. On this figure, the points related to analytical results are taken from Table 2 . On this figure, some vertices can be seen on the numerical results, corresponding to the cases when the increasing boundary at Dirichlet $\mathrm{BC}$ corresponds exactly to the passage by a vertex of the square. The results show again that both methods produce satisfying results.

\section{Comparison with other $\mathrm{BC}$ and consequences}

\subsection{General features of the results related to different boundary} conditions

These results complete the previous works concerning, Dirichlet, Neumann and Robin BC. They are synthesized in Table 3.

The evolution of the degenerate scale factor for the exterior problem with Robin $\mathrm{BC}$ and with mixed BC is similar. When $t$ decreases, the Robin BC becomes closer to a Neumann BC and when the part submitted to the Neumann BC increases, the mixed BC problem becomes also closer to a Neumann BC problem. In both cases, the degenerate scale factor increases.

\subsection{Consequences on the implementation of the BEM for plane problems}

The existence of degenerate scales for the Laplace problem and Dirichlet boundary conditions is well known since a long time. However, as seen in the previous sections, degenerate scales appear also in the case of Robin and mixed BC, the degenerate scale factors resting on a large infinite range between 1 and infinity (starting from the domain at the degenerate scale for Dirichlet BC).

The customary means to avoid the occurrence of degenerate scale when solving Laplace equation within a given domain bounded by $\Gamma$ is to use a Green's function having the form

$G_{a}=\frac{1}{2 \pi} \ln \left(\frac{a}{r}\right)$,

where $r=\|x-y\|$ and $a$ is a large positive constant. It has been shown in [69] for exterior problems, that the solution obtained by using a large value of $a$ produces an approximate solution of the 3D problem at the vicinity of a long cylinder having a length $2 a$ and a section built on $\Gamma$, which explains why this procedure is physically sound.

The background on degenerate scales in the case of Dirichlet BC allows to justify this procedure and also to give a bound on $a$ to ensure that degenerate scales do not appear when studying a problem related to a given boundary $\Gamma$. The degenerate scale factor for Laplace equation complies with the inequality

$\rho_{0} \geq \frac{2}{d_{\Gamma}}$,

where $d_{\Gamma}$ is the diameter of a circle which contains the boundary $\Gamma$. As a consequence, the degenerate scale does not appear for Dirichlet BC as soon as $a>d_{\Gamma} / 2$. With this condition, the degenerate scale factor for Dirichlet equation complies with $\rho_{0}>1$.

Looking now for Robin and mixed $\mathrm{BC}$, it has been shown that $\rho_{O(R)}>\rho_{O(D)}$ and $\rho_{O(M)}>\rho_{O(D)}$. As a consequence, the degenerate scale factors for Robin and mixed BC are always greater than 1 and the domain bounded by $\Gamma$ is never at the degenerate scale for any of the previous boundary value problems. This corresponds to the experience of scientists using the BEM.

\section{Conclusion}

The present paper completes the study of the influence of the boundary conditions on the degenerate scale for Laplace problem in the plane 
by dealing with the mixed (Dirichlet and Neumann) BC. Its main contributions concerns the case of the exterior problem where it shows the existence and the uniqueness of the degenerate scale and that the degenerate scale factor increases when the part of the boundary submitted to Neumann BC increases. It has been possible to relate directly the degenerate scale for mixed BC on a segment when the conditions are the same for both sides of segment with the degenerate scale for Dirichlet BC. For special cases of contours with symmetries (circles, squares, equilateral triangles, the problem can be solved in closed form using conformal mapping and the results for mixed BC on a segment. It is worthwhile mentioning that numerous results obtained for Laplace equation can be naturally extended to other partial differential equations like those of elasticity, Helmholtz equations,...This will be the subject of a further work.

\section{Appendix A. Obtaining matrix $[B]$ in the numerical}

\section{determination of the degenerate scale used in this work}

The coefficients of matrix $[G]$ are built from a given discretization over the contour $\Gamma$ by using $K$ collocation points $x_{i}, i=1, K$ and interpolation functions $N_{j},(j=1, K)$ by the relation:

$G_{i j}=\int_{\Gamma} N_{j}(y) G\left(x_{i}, y\right) d s(y)$

$G(x, y)=\frac{1}{2 \pi} \ln \left(\frac{1}{\|x-y\|}\right)$

Using this relation, the coefficients of matrix $\left[G_{\rho}\right]$ are given by:

$G_{\rho, i j}=\int_{\rho \Gamma} N_{j}(y) G\left(x_{i}, y\right) d s(y)$

The coefficients of matrix $\left[G_{\rho}\right]$ are obtained from those of matrix $[G]$ by:

$G_{\rho ; i j}=\int_{\Gamma} N_{j}(y) G\left(\rho x_{i}, \rho y\right) d s(y)=G_{i j}-\frac{1}{2 \pi} \ln (\rho) \int_{\Gamma} N_{j}(y) d s(y)$

This leads to :

$\left[G_{\rho}\right]=[G]-\frac{1}{2 \pi} \ln (\rho)[B]$

where

$B_{i j}=\int_{\Gamma} N_{j}(y) d s(y), \forall x_{i}$

For the case of constant elements, $N_{j}$ is equal to 1 over each element and therefore $B_{i j}$ is obviously the length of element $j$. All lines of matrix $[B]$ are identical and contain the lengths of elements along the contour.

In the case of more sophisticated elements, the tedious computation of matrix $[B]$ from the expression above involving the integration of interpolation functions can be avoided by using standard BEM codes and computing $[G]$ and $\left[G_{\rho_{1}}\right]$ for an arbitrary scale factor $\rho_{1}$.

$[B]$ is given by:

$$
[B]=\frac{2 \pi}{\ln \left(\rho_{1}\right)}\left\{[G]-\left[G_{\rho_{1}}\right]\right\}
$$

\section{References}

[1] Menon G, Paulino GH, Mukherjee S. Analysis of hypersingular residual error estimates in boundary element methods for potential problems. Comput Methods App. Mech Eng 1999;173(3):449-73.

[2] Schulz H, Stein bach O. A new a posteriori error estimator in adaptive direct boundary element methods: the Dirichlet problem. Calcolo 2000;37(2):79-96.

[3] Denda M, Dong Y. A unified formulation and error estimation measure for the direct and the indirect boundary element methods in elasticity. Eng Anal Bound Elem 2001;25(7):557-64.

[4] Alarcon E, Reverter A. p-adaptative boundary elements. Int J Numer Methods Eng $1986 ; 23(5): 801-29$

[5] Wendland W, Yu D. Adaptive boundary element methods for strongly elliptic integral-equations. Numer Math 1988;53(5):539-58.

[6] Chen J-T, Chen K-H, Chen C-T. Adaptive boundary element method of time-harmonic exterior acoustics in two dimensions. Comput Methods Appl Mech Eng 2002;191(31):3331-45.
[7] Chen K-H, Chen J-T. Adaptive dual boundary element method for solving oblique incident wave passing a submerged breakwater. Comput Methods Appl Mech Eng 2006;196(13):551-65.

[8] Simpson RN, Bordas SPA, Trevelyan J, Rabczuk T. A two-dimensional isogeometric boundary element method for elastostatic analysis. Comput Methods Appl Mech Eng 2012;209212:87-100.

[9] Simpson R, Bordas S, Lian H, Trevelyan J. An isogeometric boundary element method for elastostatic analysis: $2 \mathrm{~d}$ implementation aspects. Comput Struct 2013;118:2-12.

[10] Beer G, Marussig B, Zechner J, Duenser C, Fries T-P. Isogeometric boundary element analysis with elasto-plastic inclusions. Part 1: plane problems. Comput Methods Appl Mech Eng 2016;308:552-70,

[11] Campos LS, Albuquerque EL, Wrobel LC. An ACA accelerated isogeometric element analysis of potential problems with non-uniform boundary conditions. Eng Anal Bound Elem 2017;80:108-15.

[12] Hwang WS, Hung LP, Ko CH. Non-singular boundary integral formulations for plane interior potential problems. Int J Numer Methods Eng 2002;53(7):1751-62.

[13] Hwang WS. A regularized boundary integral method in potential theory. Comput Methods Appl Mech Eng 2013;259:123-9.

[14] Jaswon MA. Integral equation methods in potential theory, I. Proc R Soc Lond 1963;A275:23-32.

[15] Jaswon MA, Symm G. Integral equation methods in potential theory and elastostatics. London: Academic Press; 1977.

[16] Kellog OD. Foundations of potential theory. New York: Dover; 1954.

[17] Tsuji M. Potential theory in modern function theory. Tokyo: Maruzen; 1959.

[18] Landkof N. Foundations of modern potential theory. Berlin: Springer; 1972.

[19] Hayes J, Kellner R. The eigenvalue problem for a pair of coupled integral equations arising in the numerical solution of Laplace's equation. SIAM J Appl Math 1972;22(3):503-13.

[20] Hsiao GC, Maccamy RC. Solution of boundary value problems by integral equations of the first kind. SIAM Rev 1973;15(4):687-705.

[21] Hsiao GC. On the stability of integral equations of the first kind with logarithmic kernels. Arch Ration Mech Anal 1986;94(2):179-92.

[22] Yan Y, Sloan IH. On integral equations of the first kind with logarithmic kernels. J Integr Equ Appl 1988;1(4):549-79.

[23] Ransford T. Potential theory in the complex plane. Cambridge: Cambridge University Press; 1995.

[24] Christiansen S. Integral equations without a unique solution can be made useful for solving some plane harmonic problems. J Inst Math Appl 1975;16:143-59.

[25] Christiansen S. On two methods for elimination of non-unique solutions of an integral equation with a logarithmic kernel. Appl Anal 1982;13:1-18.

[26] Christiansen S. Modifications of some first kind integral equations with logarithmic kernel to improve numerical conditioning. Computing 1985;34:221-42.

[27] Chen J-T, Lin S-R, Chen K-H. Degenerate scale problem when solving Laplace's equation by BEM and its treatment. Int J Numer Methods Eng 2005;62(2):233-61.

[28] Dijkstra W, Mattheij RMM. A relation between the logarithmic capacity and the condition number of the BEM matrices. Commun Numer Methods Eng 2007;23:665-80.

[29] Chen Y-Z, Lin X-Y, Wang Z-X. Numerical solution for degenerate scale problem for exterior multiply connected region. Eng Anal Bound Elem 2009;33:1316-21.

[30] Chen Y-Z, Lin X-Y. Degenerate scale problem for the Laplace equation in the multiply connected region with outer elliptic boundary. Acta Mech 2010;225:215-33.

[31] Kuo S-R, Chen J-T, Kao S-K. Linkage between the unit logarithmic capacity in the theory of complex variables and the degenerate scale in the BEM/BIEMs. Appl Math Lett 2013;26(9):929-38.

[32] Kuo S-R, Chen J-T, Lee J-W, Chen Y-W. Analytical derivation and numerical experiments of degenerate scales for regular $\mathrm{N}$-gon domains in two-dimensional Laplace problems. Appl Math Comput 2013;219:5668-83.

[33] Chen J-T, Han H, Kuo S-R, Kao S-K. Regularization methods for ill-conditioned system of the integral equation of the first kind with the logarithmic kernel. Inverse Probl Sci Eng 2014;22(7):1176-95.

[34] Chen J-T, Kuo S-R, Kao S-K, Jian J. Revisit of a degenerate scale: a semi-circular disc. J Comput Appl Math 2015;283:182-200.

[35] Chen Y-Z. Evaluation of the degenerate scale in Laplace equation by using Newton iteration method. Eng Anal Bound Elem 2017;80:105-7.

[36] Chen J-T, Lee Y-T, Chang Y-L, Jian J. A self-regularized approach for rank-deficient systems in the BEM of 2D Laplace problems. Inverse Probl Sci Eng 2017;25(1):89-113.

[37] Chen Y-Z, Wang Z-X, Lin X-Y. Numerical examination for degenerate scale problem for ellipse-shaped ring region in BIE. Int J Numer Methods Eng 2007;71(10):1208-30.

[38] Chen Y-Z. Degenerate scale problem in antiplane elasticity or Laplace equation for quadrilaterals with arbitrary configuration. Eng Anal Bound Elem 2012;36:1370-6.

[39] Chen J-T, Lee Y-T, Kuo S-R, Chen Y-W. Analytical derivation and numerical experiments of degenerate scale for an ellipse in BEM. Eng Anal Bound Elem 2012;36(9):1397-405

[40] Chen Y-Z. Closed form solution for degenerate scale problem of Joukowcki airfoil configuration in antiplane elasticity. J Mech 2013;29(4):N21-3.

[41] Chen J-T, Shen W-C. Degenerate scale for multiply connected Laplace problems. Mech Res Commun 2007;34:67-77.

[42] Heise U. The spectra of some integral operators for plane elastostatical boundary value problems. J Elast 1978;8(1):47-79.

[43] Constanda C. On non-unique solutions of weakly singular integral equations in plane elasticity. Q J Mech Appl Math 1994;47(2):261-8. 